\title{
Brief communication: Residence time of energy in the atmosphere
}

\author{
Carlos Osácar $^{1}$, Manuel Membrado ${ }^{1}$, and Amalio Fernández-Pacheco ${ }^{1,2}$ \\ ${ }^{1}$ Facultad de Ciencias, Universidad de Zaragoza, 50009 Zaragoza, Spain \\ ${ }^{2}$ BIFI, Instituto de Biocomputación y Física de Sistemas Complejos, Plaza de San Francisco, 50009 Zaragoza, Spain
}

Correspondence: Carlos Osácar (cosacar@unizar.es)

Received: 3 October 2019 - Discussion started: 14 October 2019

Revised: 11 February 2020 - Accepted: 17 March 2020 - Published: 17 April 2020

\begin{abstract}
In atmospheric chemistry, a parameter called residence time is defined for each gas as $T=M / F$, where $M$ represents the mass of the gas in the atmosphere and $F$ is the total average influx or outflux, which in time averages are equal. In this brief communication, we extend this concept from matter to energy which is also a conservative quantity and estimate the average residence time of energy in the atmosphere, which amounts to about $58 \mathrm{~d}$. A similar estimation for the residence time of energy in the Sun is of the order of $10^{7}$ years, which agrees with the Kelvin-Helmholtz timescale.
\end{abstract}

\section{Introduction}

When the inflow, $F$, of any substance into a box is equal to the outflow, then the amount of that substance in the box, $M$, is constant. This constitutes an equilibrium or steady state. Then the ratio of the stock in the box to the flow rate (in or out) is called residence time and is a timescale for the transport of the substance in the box

$\tau=\frac{M}{F}$.

We are referring to a measurable and conserved substance. A good example of this type is the parameter defined in atmospheric chemistry as the average residence time of each individual gas, defined as Eq. (1). $M$ is the total average mass of that gas in the atmosphere and $F$ the total average influx or outflux, which in time averages for the whole atmosphere are equal. See, for example, Hobbs (2000).

In this brief communication, we want to extend the substance that flows from matter to energy and estimate the av- erage residence time of energy in the atmosphere. At the end of this brief communication, we will briefly analyze this concept for the Sun. Obviously in Eq. (1), $M$ and $F$ will now represent the total amount of energy in these two systems and the energy flux in or out, respectively.

Both cases correspond to steady-state problems because the storage of energy in the Earth's atmosphere and in the Sun is not systematically increasing or decreasing.

In Sect. 2, we consider the Earth's atmosphere as a big box, and using the appropriate energy data in Eq. (1) we compute the residence time.

In Sect. 3, we estimate the residence time of energy in the Sun and note that this residence time agrees with the KelvinHelmholtz (K-H) timescale. In Sect. 4 we present a conclusion.

\section{Forms of energy in the atmosphere and residence time}

In this section we will use the energy data provided by Hartmann (1994). The most important forms of energy in the atmosphere are the following: the thermodynamic internal energy, $U$, the potential energy due to Earth's gravity, $P$, the kinetic energy, $K$, the latent energy, $L$, related to the phase transitions of water and $E$, the total energy. The values quoted by this author of energy per unit surface in units of $10^{6} \mathrm{~J} \mathrm{~m}^{-2}$ are

$U=1800, P=700, K=1.3, L=70, E=2571$.

For our purposes of computing the residence time using Eq. (1), now we only need the inputs, and outputs, of energy in the atmosphere. It is common to express them in units of 
$e=3.42 \mathrm{~W} \mathrm{~m}^{-2}$, where $e$ is a percentage of the solar irradiance out of the atmosphere. The atmosphere absorbs $20 e$ of solar energy, $29 e$ are absorbed at the surface as sensible and latent heats, and finally it absorbs $100 e$ as long-wave radiation emitted by surface (the radiation emitted by surface is $110 e$, but $10 e$ escapes to the space using the so-called atmospheric window). Thus the total energy input in the atmosphere is

$F_{i}=20 e+29 e+100 e=149 e=509.6 \mathrm{~W} \mathrm{~m}^{-2}$.

Regarding to the emitted energy flux, we identify two terms: the component emitted spaceward, $60 e$, and that emitted to the surface, commonly denoted as the greenhouse effect, $89 e$.

We have not considered any anthropogenic contribution because it is negligible compared to the fluxes of solar origin mentioned above. In recent years, the consumption of fossil fuels has been about 10 Gtoe per year; this implies an energy flux of $0.08 \mathrm{~W} \mathrm{~m}^{-2}$ (Houghton, 2004). Then, the sum of the outgoing terms coincides with that of the ingoing terms, $F_{\mathrm{o}}=F_{\mathrm{i}}=F$. Thus, using these values of $E$ and $F$, the estimation of the residence time of energy in our atmosphere, $t_{\mathrm{a}}$ is

$t_{\mathrm{a}}=\frac{E}{F}=\frac{2571 \times 10^{6} \mathrm{~J} \mathrm{~m}^{-2}}{509.6 \mathrm{Wm}^{-2}}=5.05 \times 10^{6} \mathrm{~s} \approx 58 \mathrm{~d}$.

\section{Estimation of the solar energy and the residence time of energy in the Sun}

In stars like the Sun, the total energy, $E$, is the sum of the gravitational energy, $E_{\mathrm{g}}$, and the thermal energy, $E_{\mathrm{t}}$,

$E=E_{\mathrm{g}}+E_{\mathrm{t}}$.

The virial theorem (Kippenhan and Weigert, 1994) links the two energy reservoirs:

$-E_{\mathrm{g}}=2 E_{\mathrm{t}}$.

Therefore

$E=\frac{1}{2} E_{\mathrm{g}}$.

Using the data from Zombeck (1990), the Sun's gravitational energy can be easily estimated:

$E_{\mathrm{g}} \approx-\frac{G M_{\odot}^{2}}{2 R_{\odot}}=-1.89 \times 10^{41} \mathrm{~J}$.

Inserting (8) into (7) we obtain

$\|E\|=9.5 \times 10^{40} \mathrm{~J}$.

The ratio between $\|E\|$ and the solar luminosity, $L(3.9 \times$ $10^{26} \mathrm{~W}$ ), which constitutes the outgoing energy flux, is our estimation for the energy residence time in the Sun, $t_{\odot}$ :

$t_{\odot}=\frac{\|E\|}{L} \approx 2.6 \times 10^{14} \mathrm{~s} \approx 0.83 \times 10^{7}$ years.
The Kelvin-Helmholtz $(\mathrm{K}-\mathrm{H})$ timescale for the Sun is

$t_{\mathrm{KH}} \approx \frac{G M_{\odot}^{2}}{R_{\odot} L}$ years $=3 \times 10^{7}$ years $\approx \frac{E_{\mathrm{g}}}{L}$,

which is of the order of magnitude of the residence time of energy in the Sun $t_{\odot}$.

This timescale roughly predicts the time needed by the star to settle to equilibrium after a global thermal perturbation (Kippenhan and Weigert, 1994; Spruit, 2000; Stix, 2003). ${ }^{1}$

\section{Conclusions}

In this brief communication, we have considered our atmosphere as a big box where energy is in equilibrium and have estimated its residence time. It amounts to about $58 \mathrm{~d}$. When the same idea is applied to the Sun, we obtain $t \approx$ $0.83 \times 10^{7}$ years.

In astrophysics, the question of "how long a photon might take to get from the core of the Sun to the surface" has been frequently put forward. The answer of several authors was $\approx 10^{4}$ years (e.g., Shu, 1982; Bahcall, 1989). In 1989, Mihalas and Sills (1992) pointed out that the average step length assumed for a photon diffusing through the Sun by the previous authors was too long. Correcting this step length, they obtained $1.7 \times 10^{5}$ years. Finally, Stix (2003), invoking the large heat capacity of the interior of the star, corrected the previous result up to a timescale of the order of $10^{7}$ years. Thus, this author showed the agreement between the thermal adjustment timescale and the photon diffusion timescale.

Bearing in mind what has been said, our conclusion for the residence time of energy in Earth's atmosphere $(t \approx 58 \mathrm{~d})$ is that it is the equivalent of what the $\mathrm{K}-\mathrm{H}$ timescale is for the Sun. Therefore, after a global thermal perturbation, the atmosphere would need about a couple of months to come back to a new equilibrium.

Data availability. The data used for the estimation of residence time in the Earth's atmosphere were extracted from Hartmann (1994). The data used in the estimation of the residence time in the Sun were obtained from Zombeck (1990).

Author contributions. AFP conceived the idea; CO and AFP wrote the paper; MM contributed to the solar part.

Competing interests. The authors declare that they have no conflict of interest.

\footnotetext{
${ }^{1}$ The $\mathrm{K}-\mathrm{H}$ scale was originally proposed as an estimation of the lifetime of the Sun. This would correspond to interpreting $\tau$ in Eq. (1) as the time of depletion of an amount of energy $M$ of the box.
} 
Review statement. This paper was edited by Zoltan Toth and reviewed by two anonymous referees.

\section{References}

Bahcall, J. N.: Neutrino Astrophysics, Cambridge University Press, 1989.

Hartmann, D. L.: Global Physics Climatology, Academic Press, 1994.

Hobbs, P.: Introduction to Atmospheric Chemistry, Cambridge University Press, second edn., 2000.

Houghton, J.: Global Warming. The complete briefing 4th Edition, Cambridge University Press, 2004.
Kippenhan, R. and Weigert, A.: Stellar Structure and Evolution, Springer Verlag, 1994.

Mihalas, R. and Sills, K.: On the photon diffusion time scale for the sun, Astrophys. J., 401, 759-761, 1992.

Shu, F. H.: The Physical Universe: An Introduction to the Astronomy, Springer Verlag, 1982.

Spruit, H.: Theory of solar irradiance variations, Space Sci. Rev., 94, 113-126, https://doi.org/10.1023/A:1026742519353, 2000.

Stix, M.: On the time scale of the energy transport in the Sun, Solar Phys., 212, 3-6, 2003.

Zombeck, M. V.: Handbook of Space Astronomy and Astrophysics, Cambridge University Press, available at: http://ads.harvard.edu/ books/hsaa/ (last access: 13 April 2020), 1990. 